\title{
Detection of Abnormal High-Frequency Components in the QRS Complex by the Wavelet Transform in Patients With Idiopathic Dilated Cardiomyopathy
}

\author{
Kazuhira Maehara, MD; Toshinori Kokubun, MD; Naoyuki Awano, MD; \\ Kayano Taira, MD; Masahiro Ono, MD; Tetsuo Furukawa, MD; \\ Yoshio Shimizu, $\mathrm{PhD} *$; Yukio Maruyama, $\mathrm{MD}$
}

\begin{abstract}
In order to investigate whether increased fine, fractionated signals within the QRS complex can detect arrhythmogenic substrates and how these fine signals link with ventricular mechanical dysfunction, wavelet analysis was performed on averaged QRS complexes obtained from the left precordial lead in 26 patients with idiopatic dilated cardiomyopathy (IDCM) and in 12 normal subjects. The number of local maxima and the duration of the wavelet transform were significantly greater in patients with IDCM than in normal subjects; the number at 100 $\mathrm{Hz}$ was $8.8 \pm 3.1$ vs $6.0 \pm 1.1$ ( $\mathrm{p}<0.01)$, and the duration at $100 \mathrm{~Hz}$ was $93 \pm 15$ vs $75 \pm 7 \mathrm{~ms}(\mathrm{p}<0.01)$. Both of these indices were greater in the patients with than in those without late potentials, repetitive ventricular premature beats or cardiac death. In addition, significant inverse curvilinear relationships were observed between the left ventricular ejection fraction and both the number of local maxima and the duration of the wavelet transform. In conclusion, fine fragmented signals in the QRS complex detected by wavelet analysis would be an important marker for potentially arrhythmogenic substrates and seemed to progress in parallel with left ventricular mechanical dysfunction in IDCM. (Jpn Circ J 1999; 63: 25-32)
\end{abstract}

Key Words: Cardiomyopathy; Signal averaged electrocardiogram; Tachyarrhythmia; Ventricular pump function

$\mathbf{L}$

eft ventricular mechanical dysfunction estimated by ejection fraction (EF) and complex ventricular premature beats are independent prognostic predictors in patients with idiopathic dilated cardiomyopathy (IDCM) $!^{-4}$ From the histological viewpoint, it is likely that myocardial fiber degeneration and fibrotic replacement, resulting in the disruption of cell-to-cell connections, 5,6 would not only reduce left ventricular mechanical performance but would also produce electrophysiological abnormalities, such as disruptions of the depolarization wavefront and regional conduction delays, that would provide an appropriate substrate for arrhythmogenicity. The incidence of sudden cardiac death presumably due to fatal ventricular tachyarrhythmia increases with the progression of heart failure and the reduction of the left ventricular $\mathrm{EF}^{7,8}$ which suggests that the potential for fatal ventricular arrhythmia due to electrophysiological derangement may progress insidiously with left ventricular mechanical dysfunction, whether or not there is a history of symptomatic malignant ventricular tachycardia.

Disruptions of the depolarization wavefront and regional conduction delays, which could provide a substrate for a reentrant pathway and subsequent ventricular tachyarrhythmia, produce fine, fragmented signals in the QRS complex

(Received July 1, 1998; revised manuscript received September 21, 1998; accepted October 7, 1998)

First Department of Internal Medicine, Fukushima Medical University, Fukushima, and *Sendai National College of Technology, Sendai, Japan

Mailing address: Yukio Maruyama, MD, Professor and Chairman, First Department of Internal Medicine, Fukushima Medical University, Hikarigaoka-1, Fukushima city, Fukushima 960-12, Japan. E-mail: maruyama@cc.fmu.ac.jp of the electrocardiogram (ECG) ${ }^{9-11}$ Fine, delayed, fragmented signals in the terminal QRS/ST segment of the signal-averaged ECG, which are defined as late potentials, have been related to slowly activated tissue responsible for sustained ventricular arrhythmia and sudden cardiac death. However, late potentials are only some of the pathological signals that extend into the terminal QRS/ST segment; others might be hidden in the QRS complex, ${ }^{12}$ and the presence of late potentials has not been thought to indicate a decrease in the left ventricular ejection fraction in patients with IDCM! $1,9,13,14$

Wavelet analysis is a new time-frequency technique that enables the detection and localization of small, transient signals in the QRS complex $!^{15-22}$ Thus, this study was conducted to clarify whether fine, fragmented signals in the QRS complex detected by the wavelet transform are related to the severity of ventricular arrhythmia, cardiac mortality, the appearance of late potentials, and left ventricular mechanical dysfunction in patients with IDCM. For this purpose, we modified the original equation of wavelet analysis for a quantification of fine fragmented signals, and the relations of 4 quantitative indices; that is, the number and the density of local maxima, and the duration and the root mean square amplitude of wavelet transform, derived from wavelet transform to these issues, were evaluated.

\section{Methods}

\section{Subjects}

This study involved 26 Japanese subjects with IDCM, identified according to criteria of the World Health Organization? ${ }^{23}$ All were in sinus rhythm without bundle branch or fascicular block, or any symptoms at rest. The 
average age of the 21 males and 5 females was $52 \pm 14$ years (range, 17-68 years). Cardiac catheterization study including selective coronary angiography, left ventriculography and left ventricular endomyocardial biopsy, 24-h Holter monitoring, and wavelet analysis were performed within 2 weeks of admission.

Eleven patients were taking antiarrhythmic drugs on admission. These drugs were withdrawn at least 5 times of the half-life of each drug before Holter monitoring and wavelet analysis.

The reference group consisted of 12 apparently healthy male volunteers. Their mean age was $42 \pm 13$ years (range, 25-55 years), not significantly different from the age of the patients with IDCM.

\section{Wavelet Analysis}

The ECG was recorded from 6 unipolar left precordial leads ( 3 rows and 2 columns) including V5 and V6 leads, using a high-precision amplifier (NEC-Sanei Co, Model 1A98, Tokyo, Japan) in an electrically shielded room. Findings were stored on magnetic tape (TEAC, SR-90, Tokyo, Japan) in frequency bands from DC to $1000 \mathrm{~Hz}$. The electrocardiographic signal from the V5 lead was digitized in real time at a sampling rate of $1000 \mathrm{~Hz}$, and 10 beats were averaged using a signal processor (NEC-Sanei Co). The resolution of analog-to-digital conversion was 12 bits. The wavelet transform obtained from the $\mathrm{V}_{5}$ lead was analyzed and compared with left ventricular pump function. (The V5 lead is presumed to sensitively detect pathological signals in close proximity to the left ventricle.)

For the wavelet analysis, 10 successive QRS complexes were averaged. In a preliminary study, the ECG wavelet transform of one beat had more peaks in the higher frequency range, reflecting original ECG noise. However, no significant differences were found between 10 and 100 beats for the averaged ECG wavelet transform. We selected 10 consecutive sinus beats following at least 10 consecutive sinus beats from the stored ECG for wavelet analysis. Digitized and averaged data were stored on a floppy disc and fed to a personal computer for wavelet analysis (see Appendix), and the duration and the amplitude of the averaged QRS complex of the V5 lead were measured.

Wavelet transforms were obtained every $20 \mathrm{~Hz}$ from 20 to $220 \mathrm{~Hz}$ of the central frequency of the analyzing wavelet (see Fig 5 in Appendix). Four indices were defined for quantifying the wavelet transform at each central frequency of the analyzing wavelet: (1) the number of local maxima with amplitude in both ascending and descending limbs greater than $4 \mu \mathrm{V}$, which is an index representing the degree of fragmentation of the wavelet transform; (2) the duration from the first to the last local maximum of the wavelet transform; (3) the root mean square amplitude of the wavelet transform at each frequency for the duration defined earlier; which is an index reflecting the power spectral density of each frequency; and (4) the ratio of the number of local maxima to the duration, which indicates the density of local maxima.

\section{Detection of Late Potentials}

In order to examine how the wavelet transform obtained from the $\mathrm{V}_{5}$ lead detects late potentials, a time-domain analysis was performed using a commercially available ECG analyzer (Fukuda Denshi Co, VCM-3000, Tokyo, Japan). Briefly, bipolar pseudoorthogonal X, Y, and Z leads were obtained. The sampling rate was $1000 \mathrm{~Hz}$, and the resolution was 12 bits. Signals were averaged for 250 beats, excluding ventricular premature beats. This analysis was based on bidirectional band-pass filtering $(40-300 \mathrm{~Hz})$ and on analysis of the vector magnitude of the filtered QRS complex, according to the method of Simson et al? Late potentials were considered to be present if 2 of following 3 variables were noted: (1) filtered QRS duration $>114 \mathrm{~ms}$; (2) duration of low-amplitude signals less than $40 \mu \mathrm{V}>38$ $\mathrm{ms}$; and (3) the root mean square voltage of the last $40 \mathrm{~ms}$ of the QRS complex $<20 \mu \mathrm{V} 24$

\section{Left Ventricular Function}

Left ventricular volume in end-diastole and end-systole was measured by the area-length method at the right anterior oblique projection. The left ventricular EF was calculated by the following equation; $100 \times($ left ventricular enddiastolic volume-left ventricular end-systolic volume)/left ventricular end-diastolic volume.

Cardiac output was measured by the thermodilution method, using a Swan-Ganz catheter.

\section{Statistical Analysis}

Data were expressed as mean \pm standard deviation. The 2-tailed unpaired t test was used for comparisons between 2 groups. For comparisons among the 3 groups (ie, 2 subgroups of IDCM patients, with/without repetitive ventricular premature beats, ventricular late potentials or cardiac death, and normal subjects) 1-way analysis of variance was used, followed by Fisher's least significant difference method used as a post-hoc test. The comparisons of rate of repetitive ventricular premature beats, ventricular late potentials or cardiac death between the 2 subgroups in patients with IDCM was analyzed using a $2 \times 2$ contingency table and Fisher's direct method. Relationships between the indices of the wavelet analysis and left ventricular pump function were analyzed by the second-order polynominal regression analysis. A level of $\mathrm{p}<0.05$ was regarded as statistically significant.

\section{Results}

\section{Patient Characteristics}

All patients were in a chronic stable state at the time of study. Nine of the 26 patients were in New York Heart Association functional class I, 9 patients were in class II, and 8 patients were in class III. All patients had ventricular premature beats; the number was 5-46, 252 (mean, 3416 \pm 9346) during 24-h Holter monitoring. Ventricular premature beats were Lown grade I in 3 patients, II in 2 patients, III in 1 patient, IVa (couplets) in 3 patients, and IVb $(\geq 3$ successive beats) in the remaining 17 patients. Only 1 patient had a history of symptomatic sustained ventricular tachycardia.

Left ventriculograms revealed diffuse hypokinesis without regional wall motion abnormalities, and the coronary artery was intact or nearly intact ( $<25 \%$ stenosis) in all patients.

The left ventricular end-diastolic volume index was $140 \pm 63 \mathrm{ml} / \mathrm{m}^{2}$, and left ventricular end-systolic volume index was $99 \pm 57 \mathrm{ml} / \mathrm{m}^{2}$. The left ventricular $\mathrm{EF}$ was $30 \pm 11 \%$ (range, $13-49 \%$ ). The left ventricular peak systolic pressure and end-diastolic pressure were $119 \pm 17$ $\mathrm{mmHg}$ and $16 \pm 8 \mathrm{mmHg}$, respectively. The average cardiac index was $2.7 \pm 0.8 \mathrm{~L} \mathrm{~min}^{-1} \mathrm{~m}^{-2}$, and the heart rate at the catheterization study was $76 \pm 14$ beats/min. 
Normal, 41y, male

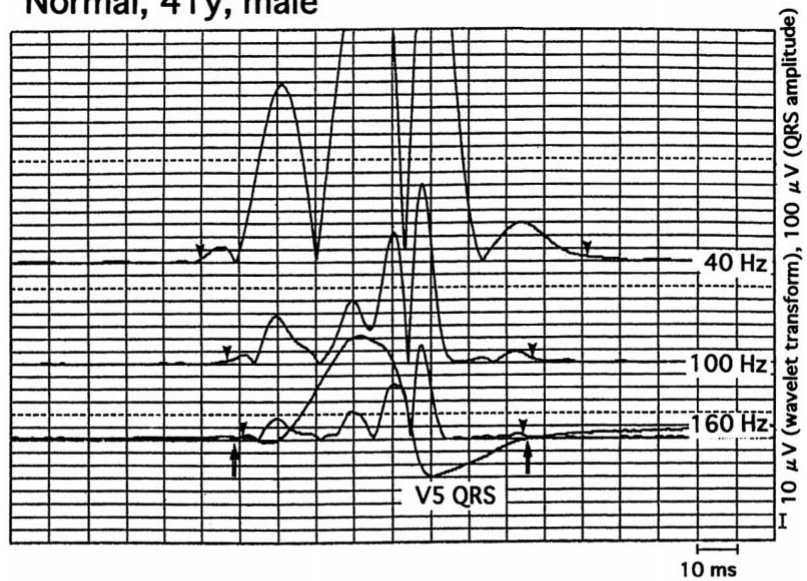

IDCM, 45y, male

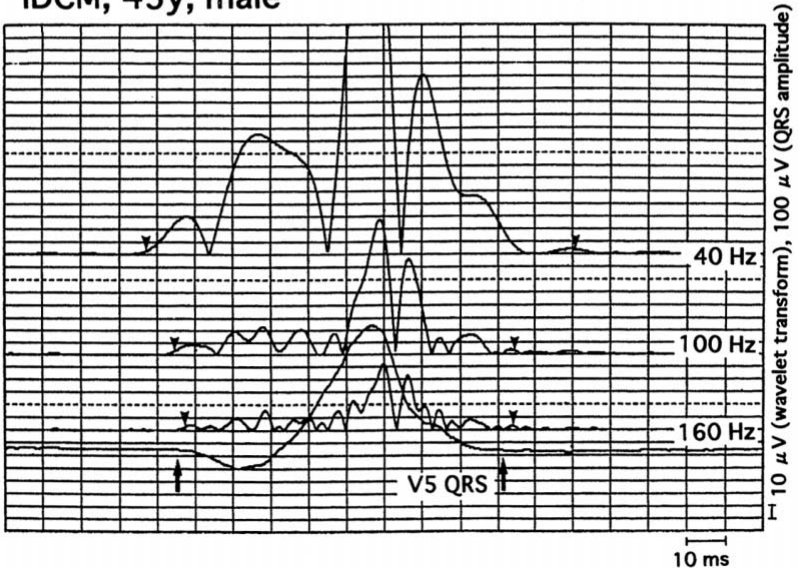

Fig 1. Averaged QRS complex obtained from precordial V5 electrocardiogram and wavelet transform at 40,100 and $160 \mathrm{~Hz}$ of the central frequency of the analyzing wavelet in a normal subject (upper panel) and a patient with idiopathic dilated cardiomyopathy (IDCM, lower panel). Arrows ( $\vee$ ) indicate the points at $4 \mu \mathrm{V}$ on the first and the last local maximum. The duration between these 2 arrows was defined as the duration of the wavelet transform. A peak with amplitude equal to or greater than $4 \mu \mathrm{V}$ in both ascending and descending limbs between the two arrows was defined as the local maximum. The QRS duration of the V5 lead is shown by the 2 markers ( $\uparrow$ ).

The follow-up period averaged $16 \pm 5$ months (range, 4-28 months). During this time, 1 patient died suddenly, and 3 patients died from progressive heart failure.

\section{Wavelet Transform in Patients With IDCM vs Normal Subjects}

The duration of averaged QRS complex of the V5 lead in patients with IDCM was $105 \pm 14 \mathrm{~ms}$, which was significantly longer than that in normal subjects $(84 \pm 7 \mathrm{~ms}$, $\mathrm{p}<0.01)$. The $\mathrm{QRS}$ amplitude was not different between the 2 groups $(1335 \pm 244 \mu \mathrm{V}$ in IDCM vs $1519 \pm 282 \mu \mathrm{V}$ in normal subjects).

Fig 1 compares representative wavelet transforms at 40, 100 and $160 \mathrm{~Hz}$ obtained from a normal subject and a patient with IDCM. In both cases, the higher the central frequency of the analyzing wavelet, the shorter was the duration and the larger was the number of local maxima in the wavelet transform. In the normal subject, there were local maxima with high amplitudes in the vicinity of the peak of the $\mathrm{R}$ wave and the nadir of the $\mathrm{S}$ wave, and a few
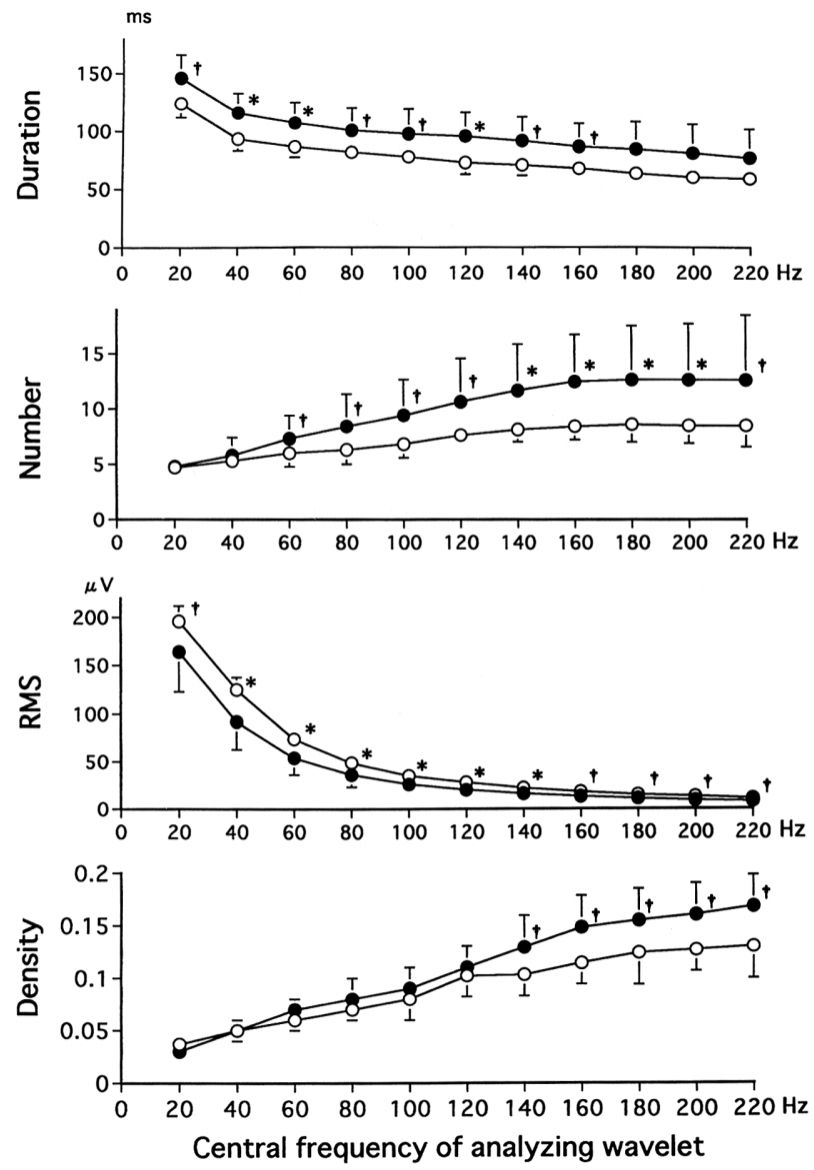

Fig 2. Duration (upper panel), number of local maxima (second panel), root mean square (RMS) amplitude (third panel) and ratio of the number of local maxima to the duration (density) of wavelet transform (lower panel) from 20 to $220 \mathrm{~Hz}$ in patients with IDCM (closed circles) and normal subjects (open circles). ${ }^{*} \mathrm{p}<0.01$ and ${ }^{\dagger} \mathrm{p}<0.05$ vs at the same frequency.

small peaks were seen in ascending and descending limbs of the QRS complex at the higher frequencies. In contrast, multiple peaks with low amplitudes were observed rather homogeneously and more densely in the wavelet transform at the higher frequencies in the patient with IDCM.

Fig 2 and Table 1 compare pooled data on the duration, number of local maxima, root mean square amplitude, and the density of local maxima of the wavelet transform between patients with IDCM and normal subjects. The duration of the wavelet transform was significantly longer in the frequency range from 20 to $160 \mathrm{~Hz}$, and the number of local maxima was greater in the range from 60 to $220 \mathrm{~Hz}$ in the patients with IDCM compared with the normal subjects. The density of local maxima was significantly greater in the patients with IDCM than in the normal subjects in the range from 140 to $220 \mathrm{~Hz}$, even though the root mean square amplitude was significantly lower in this frequency range.

Thus, increased local maxima were observed not only in the longer duration but also more densely in patients with IDCM.

For the following discussion, 40,100 and $160 \mathrm{~Hz}$ were arbitrarily selected for comparisons of wavelet transforms. 


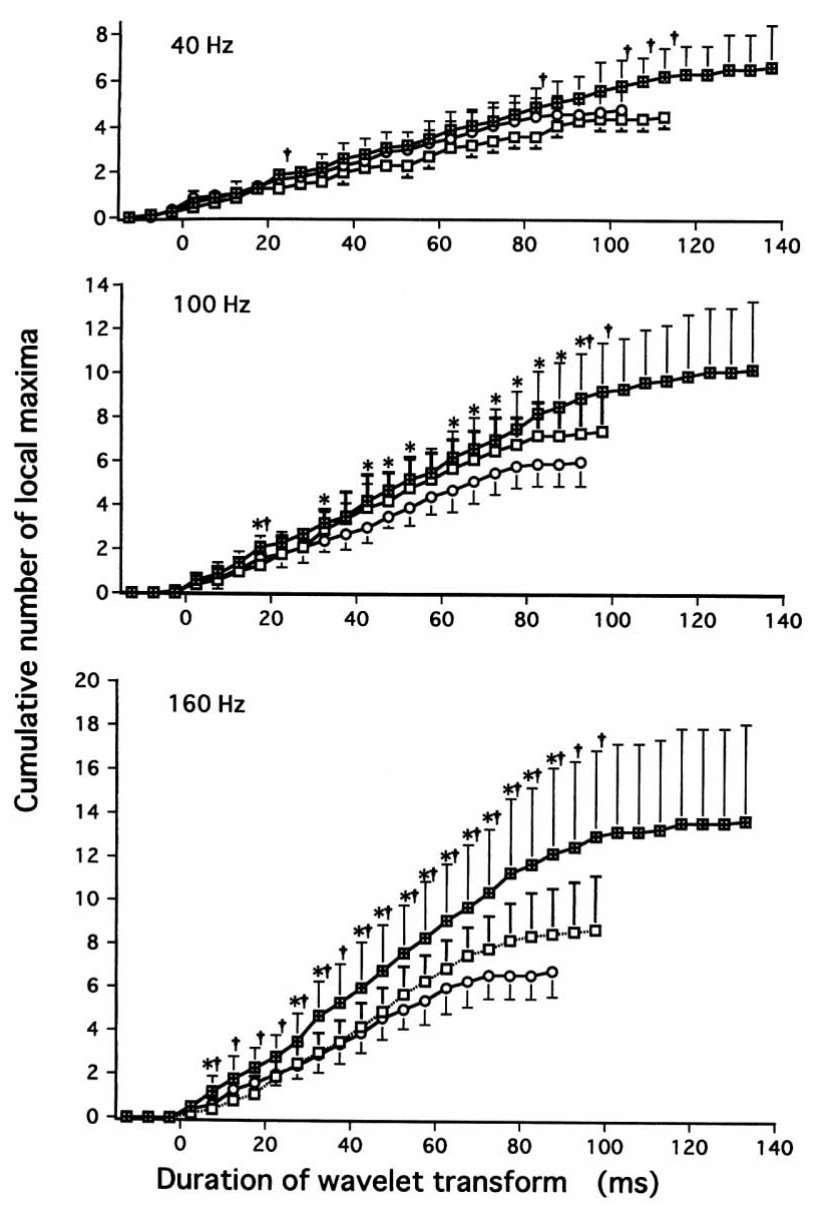

Fig 3. Cumulative numbers of local maxima at 40 (upper panel), 100 (middle panel), and $160 \mathrm{~Hz}$ (lower panel) as a function of time in IDCM patients with $(\boxplus)$ and without late potentials $(\square)$ and in normal subjects (O). The zero point on the abscissa indicates the onset of the QRS complex. The QRS duration was $111 \pm 17 \mathrm{~ms}$ in IDCM patients with late potentials, $99 \pm 10 \mathrm{~ms}$ in patients without late potentials, and $84 \pm 7 \mathrm{~ms}$ in normal subjects. Local maxima were distributed over the entire QRS complex and were present longer in IDCM patients with late potentials. ${ }^{*} \mathrm{p}<0.05$ vs normal subjects and ${ }^{\dagger} \mathrm{p}<0.05$ vs patients without late potentials.

\section{Relationship Between Wavelet Transform and Ventricular Premature Beats}

The number of ventricular premature beats in $24 \mathrm{~h}$ did not correlate with any indices of the wavelet transform at 40,100 and $160 \mathrm{~Hz}$ in the IDCM patients. However, patients with repetitive ventricular premature beats (Lown grade IV, $n=20$ ) had greater numbers of local maxima at 100 and $160 \mathrm{~Hz}$ and longer duration of the wavelet transform at 40,100 and $160 \mathrm{~Hz}$ compared with the patients without them (Lown grade I-III, $n=6$ ), as shown in Table 1 . On the other hand, the density of local maxima was not different between the 2 groups of Lown grade I-III and IV in the IDCM patients.

\section{Relationship Between Wavelet Transform and Late Potentials}

Twelve of 26 patients with IDCM had late potentials. Eleven of 12 patients with late potentials had repetitive ventricular premature beats. The QRS duration of the $\mathrm{V}_{5}$ lead was significantly longer in patients with $(111 \pm 17 \mathrm{~ms})$ than without $(99 \pm 10 \mathrm{~ms})$ late potentials $(\mathrm{p}<0.05)$ The
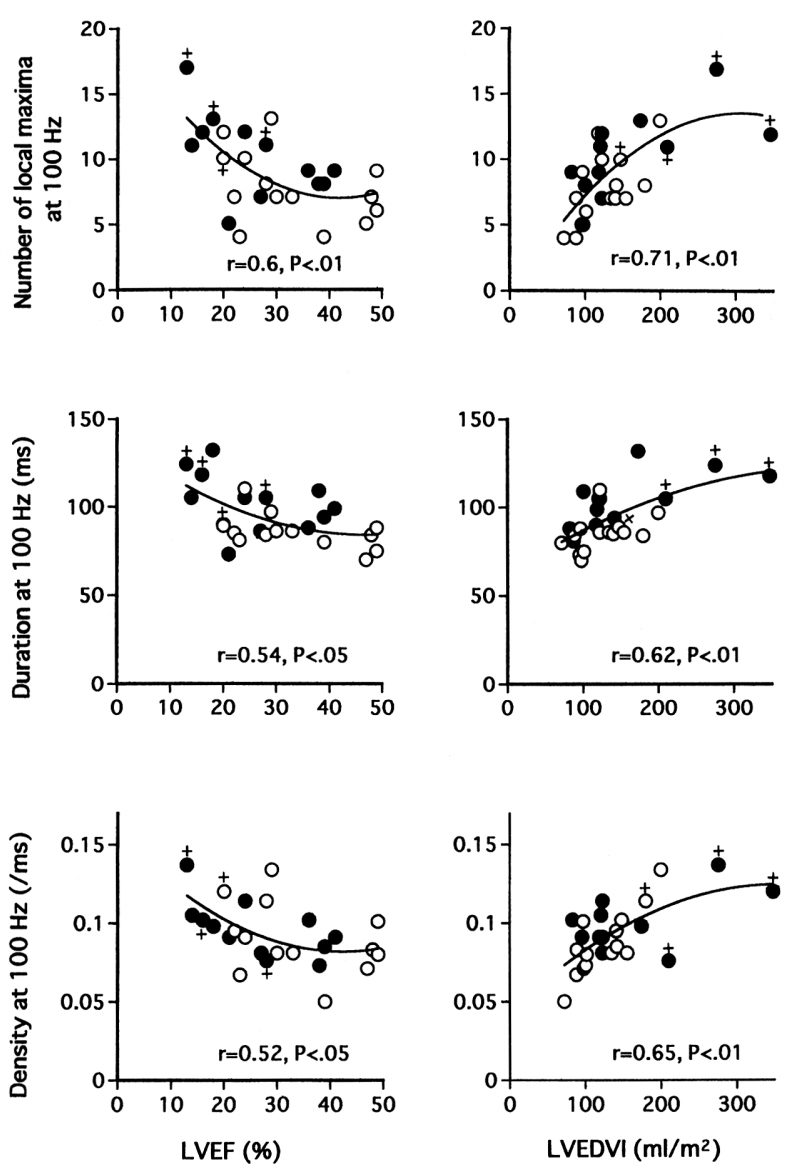

Fig 4. Correlation between parameters of left ventricular function, left ventricular ejection fraction (LVEF) and end-diastolic volume index (LVEDVI), and the number of local maxima (upper panels), the duration (middle panels) and the density of local maxima (lower panels) of the wavelet transform at $100 \mathrm{~Hz}$ in patients with IDCM. (closed circles) patients with late potentials; $(+)$ the four patients who died during the follow-up period.

filtered QRS duration of the signal-averaged vector ECG correlated with the duration of wavelet transform $(r=0.67$, $\mathrm{p}<0.05$ at $40 \mathrm{~Hz}$ ). The number of local maxima and the duration were consistently greater in patients with late potentials at 40,100 , and $160 \mathrm{~Hz}$ (Table 1 ). Fig 3 shows that number of local maxima significantly increased not only at the terminal QRS but also within the QRS complex in IDCM patients with late potentials at $160 \mathrm{~Hz}$. The duration of low amplitude signals below $40 \mu \mathrm{V}$ of signal-averaged vector ECG correlated with neither number of local maxima nor the duration of wavelet transform of whole QRS complex, but significantly correlated with the number of local maxima $(r=0.66, p<0.01$ at $160 \mathrm{~Hz})$ and the duration of wavelet transform $(\mathrm{r}=0.79, \mathrm{p}<0.01$ at $160 \mathrm{~Hz})$ after the peak of $R$ wave of the averaged V5 ECG, suggesting that wavelet transform detects late potentials. The density of local maxima was significantly greater in IDCM patients with late potentials compared with IDCM patients without late potentials and normal subjects (Table 1).

The left ventricular EF tended to be lower in patients with late potentials compared with patients without them, but the difference was not statistically significant $(27 \pm 10 \%$ vs $32 \pm 11 \%$, NS). 


\section{Relationship Between Wavelet Transform and}

Left Ventricular Mechanical Dysfunction

There were no significant relationships between root mean square amplitudes of wavelet transforms at 40, 100, and $160 \mathrm{~Hz}$ and left ventricular EF or ventricular volume indices. However, significant relationships were observed between 3 indices of the wavelet transform, the number and the density of local maxima and the duration of the wavelet transform, and left ventricular pump function in patients with IDCM (Fig 4, Table 2). Namely, there were significant positive curvilinear correlations between left ventricular end-diastolic volume index and left ventricular end-systolic volume index versus the number of local maxima, the duration of the wavelet transform at 40, 100 and $160 \mathrm{~Hz}$, and the density of local maxima at $100 \mathrm{~Hz}$. Left ventricular EF also showed significant negative curvilinear relationships with the number of local maxima, the duration of the wavelet transform at 40,100 , and $160 \mathrm{~Hz}$, and the density of local maxima at $100 \mathrm{~Hz}$.

\section{Characteristics of the Patients Experiencing}

Cardiac Death

All 4 patients who died from cardiac causes during the observation period had repetitive ventricular premature

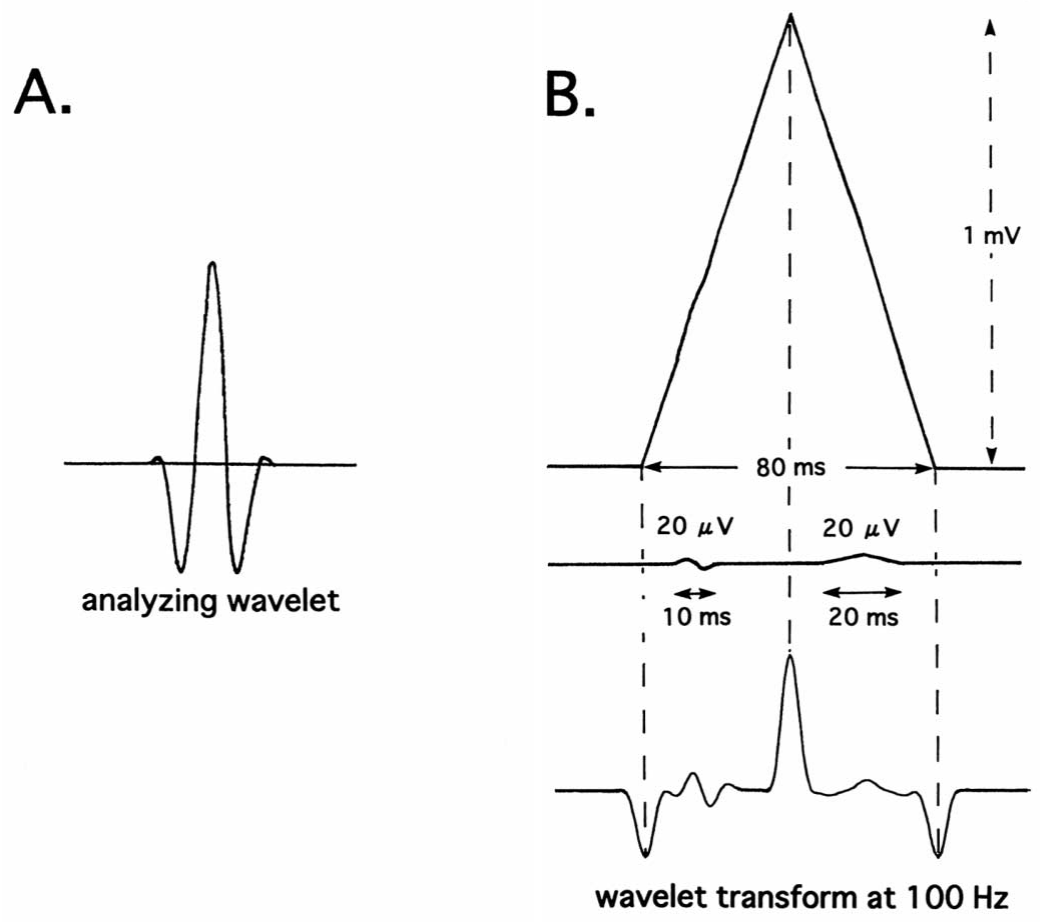

Fig 5. (A) The analyzing wavelet used in this study. The test signal (B) is composed of a large triangular wave with an amplitude of $1 \mathrm{mV}$ and a duration of $80 \mathrm{~ms}$ (which simulates the QRS complex), a sine wave with an amplitude of $20 \mu \mathrm{V}$ and a cycle length of $10 \mathrm{~ms}$ on the ascending limb, and a small triangular wave with an amplitude of $20 \mu \mathrm{V}$ and a duration of $20 \mathrm{~ms}$ on the descending limb of the large triangular wave. A small sine wave and a triangular wave simulate pathological fractionated potential within the QRS complex (middle panel). While only fine fluctuations are recognized on the ascending and descending limbs of the large triangular wave, the wavelet transform at $100 \mathrm{~Hz}$ (lower panel) definitely detects those small signals at the same temporal phase. Three peaks are also recognized on the wavelet transform at 3 points of discontinuity, corresponding to the large triangular wave.

Table 1 Three Indices of Wavelet Transform at 40, 100, and $160 \mathrm{~Hz}$ in Normal Subjects and Patients With IDCM as a Whole and in Subgroups

\begin{tabular}{|c|c|c|c|c|c|c|c|c|}
\hline & \multicolumn{2}{|c|}{ No. of local maxima } & \multicolumn{2}{|c|}{ Duration (ms) } & \multicolumn{2}{|c|}{$R M S(\mu V)$} & \multicolumn{2}{|c|}{ Density (/ms) } \\
\hline & Normal & $I D C M$ & Normal & $I D C M$ & Normal & $I D C M$ & Normal & $I D C M$ \\
\hline $40 \mathrm{~Hz}$ & $4.7 \pm 0.9$ & $5.5 \pm 1.6$ & $95 \pm 10$ & $113 \pm 16^{\dagger}$ & $125 \pm 13$ & $91 \pm 29^{\dagger}$ & $0.049 \pm 0.006$ & $0.048 \pm 0.011$ \\
\hline $100 \mathrm{~Hz}$ & $6.0 \pm 1.1$ & $8.8 \pm 3.1^{\dagger}$ & $75 \pm 7$ & $93 \pm 15^{\dagger}$ & $35 \pm 7$ & $26 \pm 10^{\dagger}$ & $0.08 \pm 0.16$ & $0.093 \pm 0.021$ \\
\hline \multirow[t]{2}{*}{$160 \mathrm{~Hz}$} & $6.8 \pm 1.2$ & $11.3 \pm 4.4^{\dagger}$ & $61 \pm 8$ & $75 \pm 20^{*}$ & $18 \pm 4$ & $13 \pm 5^{*}$ & $0.114 \pm 0.022$ & $0.148 \pm 0.031^{\S}$ \\
\hline & Lown I-III & Lown IV & LOWN I-III & Lown IV & Lown I-III & Lown IV & Lown I-III & Lown IV \\
\hline $40 \mathrm{~Hz}$ & $4.7 \pm 0.5$ & $5.8 \pm 1.8$ & $99 \pm 5$ & $118 \pm 16^{t *}$ & $96 \pm 33 *$ & $96 \pm 28^{\dagger}$ & $0.045 \pm 0.005$ & $0.049 \pm 0.012$ \\
\hline $100 \mathrm{~Hz}$ & $7.2 \pm 1.6$ & $9.4 \pm 3.3^{3+1}$ & $82 \pm 7$ & $97 \pm 15^{\dagger \neq}$ & $27 \pm 10$ & $26 \pm 10 *$ & $0.092 \pm 0.009$ & $0.093 \pm 0.023$ \\
\hline \multirow[t]{2}{*}{$160 \mathrm{~Hz}$} & $8.3 \pm 1.0^{*}$ & $12.2 \pm 4.6^{\prime+}$ & $57 \pm 11$ & $80 \pm 18^{\$ \S}$ & $14 \pm 5$ & $13 \pm 6^{*}$ & $0.148 \pm 0.021^{\dagger}$ & $0.148 \pm 0.034^{\dagger}$ \\
\hline & $L P O$ & $L P+$ & $L P O$ & $L P+$ & $L P O$ & $L P+$ & $L P O$ & $L P+$ \\
\hline $40 \mathrm{~Hz}$ & $4.4 \pm 0.5$ & $6.5 \pm 1.7 \%$ & $102 \pm 11$ & $123 \pm 14^{\dagger}$ & $88 \pm 28^{\dagger}$ & $94 \pm 32^{\dagger}$ & $0.044 \pm 0.006$ & $0.052 \pm 0.013^{\ddagger}$ \\
\hline $100 \mathrm{~Hz}$ & $7.5 \pm 2.7$ & $10.2 \pm 3.1^{\text {t⿱ }}$ & $84 \pm 7^{\dagger}$ & $102 \pm 16^{6}$ & $23 \pm 8$ & $29 \pm 11$ & $0.088 \pm 0.023$ & $0.097 \pm 0.018^{*}$ \\
\hline \multirow[t]{2}{*}{$160 \mathrm{~Hz}$} & $8.7 \pm 2.5^{*}$ & $13.7 \pm 4.4 \S$ & $65 \pm 9$ & $84 \pm 22^{\dagger+}$ & $12 \pm 4^{+}$ & $15 \pm 6^{\dagger}$ & $0.133 \pm 0.028$ & $0.162 \pm 0.028$ \\
\hline & Alive & Cardiac death & Alive & Cardiac death & Alive & Cardiac death & Alive & Cardiac death \\
\hline $40 \mathrm{~Hz}$ & $5.1 \pm 1.2$ & $7.3 \pm 2.6^{* \neq}$ & $102 \pm 17^{\dagger}$ & $122 \pm 15^{\dagger}$ & $93 \pm 28^{\dagger}$ & $86 \pm 36^{\dagger}$ & $0.046 \pm 0.008$ & $0.059 \pm 0.018^{\ddagger}$ \\
\hline $100 \mathrm{~Hz}$ & $8.3 \pm 2.7 *$ & $12.3 \pm 3.4 \$$ & $94 \pm 14^{\dagger}$ & $104 \pm 15^{\dagger}$ & $26 \pm 10^{+}$ & $27 \pm 9$ & $0.09 \pm 0.019$ & $0.109 \pm 0.026^{*}$ \\
\hline $160 \mathrm{~Hz}$ & $10.4 \pm 3.8 *$ & $15.8 \pm 5.1^{\dagger s}$ & $71 \pm 18$ & $92 \pm 19$ & $13 \pm 6^{\dagger}$ & $15 \pm 5$ & $0.144 \pm 0.031^{\dagger}$ & $0.169 \pm 0.022^{\dagger}$ \\
\hline
\end{tabular}

$L P$, late potentials; $L P 0 /+$, with/without $L P ; R M S$, root mean square amplitude of wavelet transform; ${ }^{*} p<0.05$ and ${ }^{\ddagger} p<0.01$ vs normal subjects; ${ }^{*} p<0.05$ and ${ }^{s} p<0.01$ vs comparison group at the same $\mathrm{Hz}$ in this segmental table.

Normal $(n=12)$, IDCM $(n=26)$, Lown I-III $(n=6)$, Lown IV $(n=20)$, LP O $(n=14), L P+(n=12)$, Alive $(n=22)$, Cardiac Death $(n=4)$. 
Table 2 Correlations Between Left Ventricular Mechanical Indices and Wavelet Transform in Patients With IDCM

\begin{tabular}{cccc}
\hline \hline & $\begin{array}{c}\text { LVEF } \\
(30 \pm 11 \%)\end{array}$ & $\begin{array}{c}\text { LVEDVI } \\
\left(140 \pm 63 \mathrm{ml} / \mathrm{m}^{2}\right)\end{array}$ & $\begin{array}{c}\text { LVESVI } \\
\left(99 \pm 57 \mathrm{ml}^{2} \mathrm{~m}^{2}\right)\end{array}$ \\
\hline $\begin{array}{c}\text { No. of local maxima } \\
40 \mathrm{~Hz}\end{array}$ & $-0.56^{\dagger}$ & $+0.54^{\dagger}$ & $+0.51^{*}$ \\
$100 \mathrm{~Hz}$ & $-0.60^{\dagger}$ & $+0.71^{\dagger}$ & $+0.75^{\dagger}$ \\
$160 \mathrm{~Hz}$ & $-0.60^{\dagger}$ & $+0.63^{\dagger}$ & $+0.69^{\dagger}$ \\
Duration & & & \\
$40 \mathrm{~Hz}$ & $-0.52^{\dagger}$ & $+0.55^{\dagger}$ & $+0.61^{\dagger}$ \\
$100 \mathrm{~Hz}$ & $-0.54^{*}$ & $+0.62^{\dagger}$ & $+0.67^{\dagger}$ \\
$160 \mathrm{~Hz}$ & $-0.67^{*}$ & $+0.58^{\dagger}$ & $+0.65^{\dagger}$ \\
Density & & & \\
$40 \mathrm{~Hz}$ & -0.21 & +0.43 & +0.36 \\
$100 \mathrm{~Hz}$ & $-0.52^{*}$ & $+0.65^{\dagger}$ & $+0.68^{\dagger}$ \\
$160 \mathrm{~Hz}$ & -0.19 & +0.44 & +0.44 \\
\hline
\end{tabular}

LVEDVI, left ventricular end-diastolic volume index; LVESVI, left ventricular end-systolic volume index; $L V E F$, left ventricular ejection fraction; ${ }^{*} p<0.05 ;{ }^{\dagger} p<0.01 ;+$, positive curvilinear correlation; -, negative curvilinear correlation.

beats; 1 had symptomatic ventricular tachycardia. Two of the 3 patients in NYHA functional class III and 1 patient in class II had late potentials. Their left ventricular EF was significantly lower $(20 \pm 6 \%$ vs $32 \pm 11 \%, \mathrm{p}<0.05)$, and their left ventricular end-diastolic volume index $(246 \pm 86$ vs $\left.122 \pm 33 \mathrm{ml} / \mathrm{m}^{2}, \mathrm{p}<0.01\right)$ and left ventricular end-systolic volume index $\left(198 \pm 73\right.$ vs $\left.84 \pm 30 \mathrm{ml} / \mathrm{m}^{2}, \mathrm{p}<0.01\right)$ were significantly larger compared with those in the 22 surviving IDCM patients. The number of local maxima was significantly larger at 40,100 , and $160 \mathrm{~Hz}$, and the duration of the wavelet transform at $160 \mathrm{~Hz}$ was significantly longer and the density of local maxima was greater at $40 \mathrm{~Hz}$ in patients whose lives ended in cardiac death compared with surviving patients (Table 1 ).

\section{Characteristics of the Patients With Greater \\ Fragmentation and Longer Duration of \\ Wavelet Transform}

The final subset analysis distinguished the patients whose number of local maxima at $100 \mathrm{~Hz}$ was 9 or greater, or whose duration at $100 \mathrm{~Hz}$ was $89 \mathrm{~ms}$ or longer; 17 of the 26 patients (ie, 65\%) with IDCM were in this subset. Each value for discrimination was $95 \%$ confidence limit with respect to each index in normal subjects. The density of local maxima at $100 \mathrm{~Hz}$ was larger in this group compared with the other 9 patients $(0.102 \pm 0.019$ vs $0.073 \pm 0.016 / \mathrm{ms}$, $\mathrm{p}<0.001)$. Fifteen of 17 patients in this group had repetitive ventricular premature beats and 10 had late potentials. The sensitivity to repetitive ventricular premature beats tended to be greater in this subset of wavelet transform ( 15 of 20 patients) than the appearance of late potentials (11 of 20 patients). All 4 patients who died were included in this subset. Further, there were tendencies to be greater in left ventricular end-diastolic volume index $(157 \pm 69$ vs $110 \pm 33$ $\left.\mathrm{ml} / \mathrm{m}^{2}, \mathrm{p}=0.06\right)$ and left ventricular end-systolic volume index $\left(117 \pm 62\right.$ vs $\left.73 \pm 30 \mathrm{ml} / \mathrm{m}^{2}, \mathrm{p}=0.06\right)$ in these 17 patients compared with the other 9 patients.

\section{Discussion}

The major finding of this study, in which wavelet analysis was applied to IDCM, is that the density and number of local maxima and the duration from the first to the last local maximum of the wavelet transform were significantly increased compared with these parameters in normal subjects. Moreover, these indices derived from the wavelet transform were increased in patients with repetitive ventricular premature beats and with late potentials and were significantly related to objective indices of left ventricular mechanical dysfunction. These results suggest that potentially arrhythmogenic electrophysiological derangements detected by wavelet analysis link with the left ventricular mechanical dysfunction in patients with IDCM regardless of the presence of symptomatic malignant ventricular arrhythmia.

The high resolution ECG has been investigated as a potential noninvasive tool yielding useful information about electrocardiographic abnormalities. Wavelet analysis is a recently introduced time-scale technique that estimates the frequency content of a signal as a function of time.$^{15,16}$ This method has been applied to the analysis of the signalaveraged ECG, including the localization of irregularities in the QRS complex or late potentials caused by buried fractionated potentials ${ }^{16-22}$ and has been reported to identify postinfarction patients with serious ventricular arrhythmia ${ }^{20,21}$ Previous studies used a relative amplitude to define a local maximum, therefore the cutoff value of a local maximum was dependent on the greatest magnitude of wavelet transform. In the present study, the amplitude of wavelet transform was expressed as an absolute value by the modification of the original equation and had a more substantial meaning, as described in the Appendix.

Compared with other time-frequency analyses, such as short-time Fourier transform and Wigner distribution, wavelet analysis has better simultaneous time-frequency resolution and less oscillatory cross-terms ${ }^{17}$ Our study showed that high-frequency components were increased in patients with late potentials, not only at the terminal portion of the QRS complex but also within the QRS complex, indicating the increase in fragmentation within the whole QRS complex. Actually, IDCM patients with a relatively large number of local maxima and the long duration of the wavelet transform did not always have late potentials, as shown in Fig 4, and the distribution of fragmentation was not homogeneous over the whole QRS complex. The appearance rate of late potentials detected in these IDCM patients tended to be smaller than the abnormality rate estimated from the number of local maxima and duration of wavelet transform at $100 \mathrm{~Hz}[12 / 26(46 \%)$ vs $17 / 26(65 \%)$ ]. Moreover, it seemed likely that the greater fragmentation and the longer duration of wavelet transform might highly predict the occurrence of repetitive ventricular premature beats, compared with late potentials; however, a large population study would be needed for a comparison between them of the predictive power for malignant ventricular arrhythmia.

On the other hand, left ventricular dysfunction did not relate to the appearance of late potentials, as previously reported $1,13,14$ but did correlate with the degree of fragmentation of the wavelet transform. The degree of left ventricular dysfunction, reflected by objective indices and functional class, remains the most reliable and potent predictor of both total mortality and sudden death in patients with nonischemic dilated cardiomyopathy.,25 In addition, a recent multicenter study in 218 patients with IDCM revealed that cardiac mortality was significantly higher in patients with repetitive ventricular premature beats compared with patients with no or isolated ventricular 
premature beats during a follow-up period of $29 \pm 16$ months. In the present study, electrophysiological abnormalities detected by wavelet analysis correlated with left ventricular dysfunction and repetitive ventricular premature beats as well as cardiac death, although there was no relationship between left ventricular dysfunction and repetitive ventricular premature beats. Thus, fine fragmentation detected by wavelet analysis in the whole QRS complex, which would reflect the disruption of the excitation wavefront and regional conduction delays presumably caused by myocardial degeneration and fibrosis, would be a common index for arrhythmogenicity and left ventricular mechanical dysfunction in patients with IDCM.

Finally, there are some methodological limitations to be discussed. First, the cutoff amplitude of the local maximum was arbitrarily defined as $4 \mu \mathrm{V}$ in this study. Local maxima with smaller amplitudes might provide other pathophysiological information. Second, the QRS duration of the V5 lead in patients with IDCM was significantly longer than that in normal subject and therefore we also used the index normalized by the duration; that is, the density of local maxima in order to compare differences in fine fragmentation between IDCM and normal subjects. However, patients with definite bundle branch or fascicular block were excluded because they increase the QRS duration, subsequently increasing the duration of wavelet transform regardless of regional conduction disturbances, and it has been already reported that these conduction disturbances are the major independent risk factor for cardiac death in patients with dilated cardiomyopathy ${ }^{26}$ Although it is likely that wavelet analysis can discriminate the characteristics of IDCM patients more definitely in the presence of any conduction disturbances, further investigation are needed to clarify these issues.

\section{Appendix}

In general, the wavelet transform is defined as follows:

$$
\mathrm{S}(\mathrm{a}, \mathrm{b})=(1 / \sqrt{\mathrm{a}}) \int_{+\infty}^{-\infty} f(\mathrm{t}) \mathrm{g}\{(\mathrm{t}-\mathrm{b}) / \mathrm{a}\} \mathrm{dt}
$$

where $f(t)$ is the electrocardiographic signal, a is the scale $(>0)$, and $b$ is the time. By using $1 / \mathrm{a}$ instead of $1 / \sqrt{ } \mathrm{a}$, we can get a linear scale of the wavelet transform.

$$
\mathrm{S}(\mathrm{a}, \mathrm{b})=(1 / \mathrm{a}) \int_{+\infty}^{-\infty} f(\mathrm{t}) \mathrm{g}\{(\mathrm{t}-\mathrm{b}) / \mathrm{a}\} \mathrm{dt}
$$

An analyzing wavelet is used for the expansion and synthesis of the signal. The analyzing wavelet used in this study is formed by multiplying cosine waves using a Hanning window function, as shown in Fig 5A. That is,

$$
g(t)=(1 / 2) \cos 2 \pi f t(1+\cos \pi f t)
$$

The 1/a in equation (1') is $f$ in equation (2). From the preceding discussion, we get a pertinent wavelet transform, as follows:

$$
\mathrm{S}(\mathrm{f}, \mathrm{z})=(f / 2) \int f(\mathrm{t}) \cos 2 \pi f(\mathrm{t}-\mathrm{z})[1+\cos \pi f(\mathrm{t}-\mathrm{z})] \mathrm{dt}
$$

In the preliminary study using a test signal, wavelet analysis definitely detected fine transient signals hidden in the QRS complex, as shown in Fig 5B. In the actual analysis, the amplitude was expressed as an absolute value to simplify evaluation of the wavelet transform.

\section{References}

1. Mancini DM, Wong KL, Simson MB: Prognostic value of an abnormal signal-averaged electrocardiogram in patients with nonischemic congestive cardiomyopathy. Circulation 1993; 87: 1083-1092

2. De-Maria R, Gavazzi A, Baroldi G, De-Vita C, Camerini F: Comparison of clinical findings in idiopathic dilated cardiomyopathy in women versus men. Am J Cardiol 1993; 72: 580-585

3. Cianfrocca C, Pelliccia F, Nigri A, Critelli G: Resting and ambulatory ECG predictors of mode of death in dilated cardiomyopathy. $J$ Electrocardiol 1992; 25: 295-303

4. De-Maria R, Gavazzi A, Caroli A, Ometto R, Biagni A, Camerini F: Ventricular arrhythmia in dilated cardiomyopathy as an independent prognostic hallmark: Italian Multicenter Cardiomyopathy Study (SPIC) Group. Am J Cardiol 1992; 69: 1451 - 1457

5. Roberts WC, Siegel RJ, McManus BM: Idiopathic dilated cardiomyopathy: analysis of 152 necropsy patients. Am J Cardiol 1987; 80: $1340-1355$

6. Unverferth DV, Olsen EG: Extent of myocardial fibrosis and cellular hypertrophy in dilated cardiomyopathy. Am J Cardiol 1986; 57: $816-820$

7. Kjekshus J: Arrhythmia and mortality in congestive heart failure. $A m$ J Cardio 1990; 65: I42-I48

8. Califf RM, McKinnis RA, Burke J, Lee KL, Harrel FE, Behar VS, et al: Prognostic implications of ventricular arrhythmia during 24 hour ambulatory monitoring in patients undergoing cardiac catheterization for coronary artery disease. Am J Cardiol 1982; 50: 23-31

9. Simson MB, Untereker WJ, Spielman SR, Horowita LN, Marcus $\mathrm{NH}$, Falcone RA, et al: Relation between late potentials on the body surface and directly recorded fragmented electrograms in patients with ventricular tachycardia. Am J Cardiol 1983; 51: 105-112

10. Lesh MD, Spear JF, Simson MB: A computer model of the electrogram: what causes fractionation? J Electrocardiol 1988; 21(Suppl): $69-73$

11. Josephson ME, Horowitz LN, Farshidi A: Continuous local electrical activity: a mechanism of reentrant ventricular tachycardia. Circulation 1978; 57: 659-665

12. Hood MA, Pogwizd SM, Peirick J, Cain ME: Contribution of myocardium responsible for ventricular tachycardia to abnormalities detected by analysis of signal-averaged ECGs. Circulation 1992; 86: $1888-1901$

13. Poll DS, Marchlinski F, Falcone RA, Josephson ME, Simson MB: Abnormal signal averaged ECG in non-ischemic congestive cardiomyopathy: relationship to sustained ventricular arrhythmias. Circulation 1985; 72: 1308-1313

14. Keeling PJ, Kuiakowski P, Yi G, Slade AKB, Bent SE, Mckenna WJ: Usefulness of signal-averaged electrocardiogram in idiopathic cardiomyopathy for identifying patients with ventricular arrhythmias. Am J Cardiol 1993; 72: $78-84$

15. Combes JM, Grossman A, Tchamitchian P: Wavelets: timefrequency methods and phase space. Springer-Verlag, Berlin, 1989

16. Maehara K, Furukawa T, Shimizu Y, Maruyama Y: Wavelet analysis of the QRS complex of signal averaged electrocardiogram in normal men (abstract). Ann Noninvasive Electrocardiol 1996; 1: 237

17. Jones DL, Touvannas JS, Lander P, Albert DE: Advance timefrequency methods for signal-averaged ECG analysis. $J$ Electrocardiol 1992; 25(Suppl): 188-194

18. Crowe JA, Gibson NM, Woolfson MS, Somekh MG: Wavelet transform as a potential tool for ECG analysis and compression. J Biomed Eng 1992; 14: 268-272

19. Morlet D, Peyrin F, Desseigne P, Touboul P, Rubel P: Time-scale analysis of high-resolution signal averaged surface ECG using wavelet transform. In: Murray A, Arzbaecher RC, editors: Computers in cardiology. IEEE Computer Society, Los Alamitos, 1991; 393-396

20. Morlet D, Peyrin F, Desseigne P, Touboul P, Rubel P: Wavelet analysis of high resolution signal averaged ECGs in postinfarction patients. J Electrocardiol 1993; 26: 311-320

21. Reinhardt L, Makijarvi M, Fetsch T, Montonen J, Sierra G, Martinez-Rubio A, et al: Predictive value of wavelet correlation functions of signal-averaged electrocardiogram in patients after anterior versus inferior myocardial function. J Am Coll Cardiol 1996; 27: $53-59$

22. Dickhaus H, Khadra L, Brachmann J: Quantification of ECG late potentials by wavelet transformation. Comput Methods Programs Biomed 1994; 43: $185-192$

23. Brandenburg RO, Chazov E, Cherian G, Falase AO, Grosgogeat Y, Kawai C, et al: Report of WHO/ISFC task force on definition and classification of cardiomyopathies. Br Heart J 1980; 44: 672-673

24. Breihardt G, Cain ME, El-Sherif N, Flowers N, Hombach V, Janse 
M, et al: Standards for analysis of ventricular late potentials using high resolution or signal-averaged electrocardiography: A statement by a Task Force Committee between the European Society of Cardiology, the American Heart Association and the American College of Cardiology. Eur Heart J 1991; 12: 473-480

25. Turitto G, Ahuja R, Caref EB, El-Sherif N: Risk-stratification for arrhythmic events in patients with nonischemic dilated cardiomyopa- thy and NSVT: role of programmed ventricular stimulation and the signal-averaged electrocardiogram. J Am Coll Cardiol 1994; 24: $1523-1528$

26. Koga Y, Wada T, Toshima H, Akazawa K, Nose Y: Prognostic significance of electrocardiographic findings in patients with dilated cardiomyopathy. Heart Vessels 1993; 8: 37-41 